\title{
Smart Healthcare Apps for Quality Cancer Patient Support
}

Angelina Kouroubali, Institute of Computer Science, Foundation for Research and Technology-Hellas (FORTH-ICS), Greece

iD https://orcid.org/0000-0002-3023-8242

Lefteris Koumakis, Institute of Computer Science, Foundation for Research and Technology-Hellas (FORTH-ICS), Greece Haridimos Kondylakis, Institute of Computer Science, Foundation for Research and Technology-Hellas (FORTH-ICS), Greece

Dimitrios G. Katehakis, Institute of Computer Science, Foundation for Research and Technology-Hellas (FORTH-ICS), Greece

(iD https://orcid.org/0000-0002-3763-191X

\section{ABSTRACT}

This paper analyzes the clinical and technological characteristics of cancer mobile apps that enable patients to securely record, manage, and share their information. It proposes an integrated approach towards developing quality mobile health apps for cancer and discusses issues relevant to the enhancement of patient experience and acceptance, adherence to treatment, and effective support of coordinated care. Regulation, standardization, and interoperability together with the existence of useful, accurate, and reliable tools for active patient engagement are important aids towards efficient cancer disease management. Improving quality of life and well-being, in a secure and safe manner that respects the patients' privacy, seems to be a key challenge, together with the exploitation of the outcomes of recent research projects, relevant to end user digital engagement towards an integrated approach for the introduction of trustworthy, interoperable, usable, adaptable, and quality mobile health apps.

\section{KEYWORDS}

Big Data, Data Regulations, Electronic Health Record, Interoperability, Modular Architecture, Patient Data Privacy, Patient Empowerment, Personal Health Record, Quality of Life, Self-Management

This article, originally published under IGI Global's copyright on April 17, 2020 will proceed with publication as an Open Access article starting on January 18, 2021 in the gold Open Access journal, International Journal of Big Data and Analytics in Healthcare (converted to gold Open Access January 1, 2021), and will be distributed under the terms of the Creative Commons Attribution License (http://creativecommons.org/licenses/by/4.0/) which permits unrestricted use, distribution, and production in any medium, provided the author of the 


\section{INTRODUCTION}

Cancer is a generic term used to describe a large group of diseases that can affect any part of the body and is a leading cause of death worldwide (https://www.who. $\mathrm{int} /$ news-room/fact-sheets/detail/cancer). Cancer incidents have increased from 12.7 million in 2008 to 14.1 million in 2012 and 18.1 million new cases in 2018, and this trend is projected to continue (Bray et al., 2018). The different phases in the cancer care continuum are prevention, early detection, diagnosis, treatment, survivorship, and end-of-life care. Advances in medicine help patients increase their survival rate, or eventually be cured, when cancer occurs (McGuire, 2016). As cancer is perceived more as a chronic, rather than an acute disease, concepts of patient empowerment and self-management become relevant also in the oncologic field (Kushi et al., 2012).

Cancer patients require supportive care during and after treatment. These relate to physical, emotional, and social needs that vary over time and between treatments. Care and support for patients with cancer should include early recognition of signs and symptoms, support for self-care, personalized care planning, and routine use of patient-reported outcome (PRO) measures (Maher, 2013). Routine reporting of patient outcomes greatly facilitates the identification of present problems and impact of treatment. Patient-reported outcomes (PROs) also enhance patient-clinician communication that promotes shared decision-making (Kotronoulas et al., 2014; Valderas et al., 2008). Reporting of outcomes and interacting with physicians over a digital application or app has been shown to lower overall symptoms of distress, improve quality of life (QoL), and result in fewer emergency visits and improved overall survival (Basch et al., 2016; Denis et al., 2017).

Mobile devices are becoming an integral part of the healthcare industry, changing how care is delivered and received. In recent years, the Internet of Things (IoT) has emerged as a new computing paradigm, in which a continuum of devices, sensors and objects are interconnected with a variety of communication solutions. IoT can help improve the living standard of citizens. It is anticipated that more than 50 billion devices, ranging from smartphones, laptops, sensors, and game consoles, will be connected to the Internet through several heterogeneous access network technologies such as radio-frequency identification and wireless sensor networks (Triantafyllidis et al., 2016; Yaqoob et al., 2017).

Healthcare systems around the world are becoming increasingly interested in strengthening the role of patients in their care. Empowerment occurs when patients increase their capacity to think critically and make autonomous, informed decisions. In that process, the role of healthcare providers, technology providers, citizens, and policy-makers is of paramount importance. An increasing trend shows a shift of healthcare models towards prediction, early detection and monitoring. 


\section{BACKGROUND}

Mobile Health (mHealth) has revolutionized the ability of citizens to manage their own health and personal health data. Personal health record (PHR) systems facilitate the collection of information about an individual's health and health care, stored in an electronic format intended for use by consumers, patients or their informal caregivers. The electronic health record (EHR) is a more generic term and encompasses the PHR and the electronic medical record (EMR) concepts (Katehakis \& Tsiknakis, 2006). PHR systems aim to address patients' evolving needs using specific methods to improve their care and foresee health issues.

A PHR with cancer modules has been seen as a potentially useful tool for patients in cross-sector cancer care (Kondylakis et al., 2017a). It has the potential to support patients in managing their chronic illness. As a tool, it facilitates information exchange between patients and healthcare professionals as well as between healthcare professionals and/ or institutions across health care sectors. PHR adoption and use in the context of cancer is not yet widespread (Wiljer et al., 2010), however, when it is implemented, PHR use is frequent and increasing (Roehrs et al., 2017).

Despite the existence of hundreds of studies involving cancer-focused mobile apps, there is a lack of collective efforts to determine cancer-focused apps that effectively represent useful, accurate, and reliable tools for cancer disease management (Rincon et al., 2017). Safety concerns highlight the need for regulation, full authorship disclosure and clinical trials. A robust framework for identifying high-quality applications is necessary. Applications should also undergo a thorough evaluation and quality labelling procedure to ensure the quality and effective functionality of application features. Categories of criteria for apps evaluation include engagement, functionality, aesthetics, information quality, and subjective quality scale. Quality assessment scales of mobile health apps can be used to provide a checklist for the design and development of new high-quality health apps (Stoyanov et al., 2015).

As the industry shifts to digital care delivery, healthcare systems are already developing consumer-focused practices (Kouroubali et al., 1997; Kouroubali, 2004). Healthcare consumers with digital technology are now expecting custom-made service anytime and anywhere, forcing providers to develop new models of care (Cognizant, 2019). On 07.11.2019, the German Parliament passed a new law on the provision of digital healthcare to patients (https://www.hlregulation.com/2019/11/15/ digital-health-solutions-to-become-reimbursable-in-germany-milestone-for-patientsand-providers/\#page $=1$ ). According to the new legislation, compensation will be provided for digital health solutions that are low risk medical devices (Class I or IIa) with core functionality based on digital technologies (applications or mainly software based solutions). The purpose of using the solution should be to diagnose, monitor or treat illnesses or improve the health care provided. Non-medical devices or digital applications used in parallel or in combination with other medical devices are not covered. A medical benefit may not be verifiable without the collection of clinical patient data. An improvement in the structure and process of healthcare could be easier 
to prove - but still there is a need for support by real-world data. Privacy, security and confidentiality of health data managed in mHealth apps are of pivotal importance. Designers need to be able to certify that their policies satisfy the legal requisites in privacy and security (Martínez-Pérez, B. et al., 2015).

The shift from cancer as a terminal illness to cancer as a chronic illness requires that patients and their families assume a more central role in the management of the oncologic disease, healthcare professionals take a partnership with patients accepting to share responsibilities, and policymakers provide adequate support to the healthcare systems in implementing new models (McGuire, 2016). The use of PROs has become a prominent topic in healthcare innovation, highlighting the role of the patient experience as a key measure of healthcare quality (LeBlanc \& Abernethy, 2017). A PRO is defined as "a measurement based on a report that comes directly from the patient about the status of a patient's health condition without amendment or interpretation of the patient's response by a physician or anyone else" (US Food \& Drug Administration, 2006). In the past, PROs were used solely in the context of research studies, collected on paper as part of a research protocol, and were used largely as secondary or exploratory endpoints in clinical trials or observational studies. As such, these data were not typically made available to the clinical team and had no influence on patient care. Over time, PROs have taken a prominent place in oncology research. PROs are currently used regularly as secondary endpoints in oncology drug trials, and sometimes even as primary endpoints, in both the pediatric and adult population (Maramis et al. 2019). The prioritization of self-management support for cancer survivors not only builds on the evidence that patients want to be involved in healthcare decisions, but it is also grounded on a shift towards a more personalized and tailored approach to care, following cancer treatment.

Several European Union projects have been implemented for the design and development of personalized health applications for the management of cancer for patient empowerment and self-management. Some of them are briefly presented below:

- iManageCancer: "Empowering patients and strengthening self-management in cancer diseases" (http://imanagecancer.eu/, 2015-2018) provided a cancer-specific self-management platform designed according to the needs of patient groups. It focused on the wellbeing of cancer patients with special emphasis on avoiding, early detecting and managing adverse events of cancer therapy, and on the psychoemotional evaluation and self-motivation goals. In this context, developed cancer specific apps allowed patients, through an easy-to-use interface for mobile devices, to keep track of their health and disease status and to maintain a diary on personal clinical observations such as side effects of therapies that could be shared with healthcare providers;

- BOUNCE: "Predicting Effective Adaptation to Breast Cancer to Help Women to BOUNCE Back" (https://www.bounce-project.eu/, 2017-2021) delivers a unified clinical model of modifiable factors, associated with optimal disease outcomes and advanced computational tools, to be deployed for a prospective multi-center clinical 
pilot at four major oncology centers (Manikis et al., 2019). The overreaching goal of the project is to incorporate elements of a dynamic, predictive model of patient outcomes in building a decision-support system used in routine clinical practice to provide physicians and other health professionals with concrete, personalized recommendations regarding optimal psychosocial support strategies;

- MyPal: "Fostering Palliative Care of Adults and Children with Cancer through Advanced Patient Reported Outcome Systems" (https://mypal-project.eu/, 20192022) aims to foster palliative care for people with cancer by leveraging PRO systems through their adaptation to the personal needs of the person with cancer and his/ her caregiver(s). The project aspires to empower people with cancer and their caregivers in capturing more accurately their symptoms/ conditions, communicate them in a seamless and effective way to their healthcare providers and, ultimately, foster the time for action through the prompt identification of important deviations in the patient's state and QoL. Furthermore, an innovative voice-based dialogue interaction method, also known as conversational agent, will be implemented as an alternative way for the patients to interact with the MyPal platform and report PROs (Chatzimina et al 2019).

European research projects such as the ones described above offer results that provide valuable scientific insights about the appropriate modules that are necessary to enhance cancer care and promote patient empowerment and self-management practices.

\section{IMPLEMENTING SMART HEALTHCARE APPS FOR CANCER}

Research has shown that cancer patients value the use of apps for health care management and feel comfortable using them (Girault et al., 2015). Designing a user-centred, patient-focused app, tailored to the individual user needs, requires the early involvement of both patients and healthcare professionals in the development, implementation, and evaluation processes. User involvement is very important and needs to be established from the application conception. User feedback from doctors and cancer patients will help determine which apps or types of apps are most effective, and among which groups of survivors.

Cancer applications may include features for the assessment of the occurrence, frequency and distress of symptoms, together with a connection to a monitoring web interface. They may also include features such as a risk assessment model that sends alerts via text messages to health care providers, continuous access to evidencebased, self-care advice, links to relevant websites for more information, and graphs of symptom reporting history for patients and health care providers. A good app should empower patients by providing useful information that enables them to manage the challenges of their treatment (Kouroubali et al., 2019a). Self-management apps play a crucial role in helping patients to cope with their illness and facilitate communication with their healthcare professionals. 


\section{Integrated Architecture}

In order to implement a mobile platform focusing on empowering cancer patients, an integrated, modular and extensible architecture is required. A high-level, two-layer architecture facilitates the development of a multitude of interrelated apps, based on common modules. A data management layer is collecting and integrating all available data. The graphical user interface layer includes many individual apps offering three kinds of services, administration apps, core patient apps and cancer-specific apps. Generic services (admin and core patient apps), as well as cancer management services, are described first, followed by the description of the data layer that underlines them. The list of presented services is by no means complete. It can be extended with the addition of any relevant modules to support clinical and administrative needs (e.g. for insurance eligibility notification, etc.) not included here. The suggested modular architecture of a cancer mobile app is presented in Figure 1. The apps facilitate the communication and use of data among different supporters including patients, health providers, family, friends and social networks, researchers and policymakers.

Figure 1. Typical set of software modules required for cancer patient empowerment

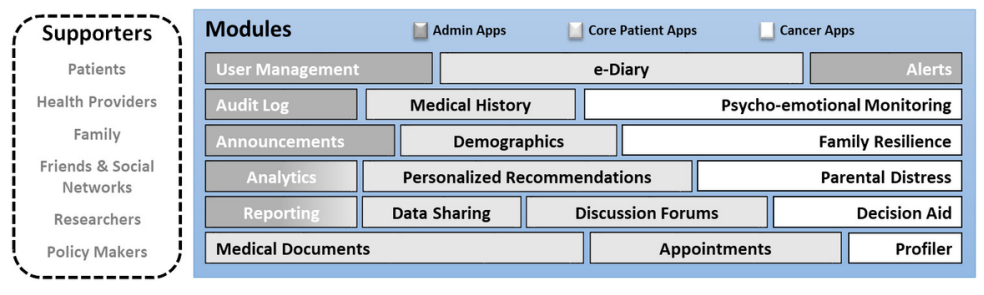

\section{Generic Services}

Generic services consist of basic health monitoring modules that should be available to support both admin and core patient services. Admin services consist of apps enabling user management (registration, complete delete data, remind password etc.), auditing, platform announcements and alerts, and apps for analyzing the available data through data mining algorithms.

Core patient services consist of apps mostly capturing demographics and medical history such as allergies, problems, procedures, laboratory results, and measurements. All medical documents such as discharge letters, laboratory results, imaging data and others should be able to be stored in one place that the individuals will be able to navigate, search and visualize. The documents app provides this functionality. In addition, an app should allow users to share selected data with other users or doctors. An e-diary offers a graphical overview of all available information, based on the appropriate profile settings, also enabling the users to make and view appointments with their doctors. An intelligent personal health recommendation app enables patients to search for health information in a high-quality document corpus selected by health 
experts. Intelligent alerts in case of drug interactions and other serious events detected are also part of such a module.

\section{Cancer-Specific Services}

A decision aid app assists patients in making informed decisions about their treatment options based on the individual patient's priorities. All collected information feeds a profiler app enabling the graphical representation of a patient profile. These services are further described below:

- Psycho-emotional Monitoring: As cancer highly affects the psycho-emotional status of individuals, standardized, validated, psycho-behavioural questionnaires should be used in order to monitor the psycho-emotional status of cancer patients and their resources in coping with cancer. Such information could feed interventions not only targeting at improving the health status of the patient but also his/ her ability to cope with the disease;

- Family Resilience: To support holistic management of cancer, tools for effective measurement of family resilience are very important. These tools investigate areas concerning crucial psychological factors within a family such as low esteem/ selfefficacy, negative thinking, emotional reactions, personal sense of competence, self-reliance, health beliefs and other factors. These factors directly relate to interpersonal relationships such as communication and problem-solving skills, family disharmony, instability or breakout and family attitudes and values;

- Parental Distress: In the same idea of holistic management as the family resilience tool, developing solutions to monitor parental distress is paramount in addressing it effectively. An example of such a tool is the Psychosocial Assessment Tool (Pai et al., 2007) designed for families of children with cancer and distinguishes among families that are at universal, targeted or clinical risk for psychosocial problems;

- Decision Aid: These tools translate evidence into a patient-friendly form by providing, at a minimum, information about the options, benefits and risks, and implicit methods to clarify personal values. These tools have the potential to facilitate patient empowerment in the decision-making process;

- Profiler: This tool enables doctors to easily search for a specific patient and visualize results, providing appropriate recommendations.

\section{Data Layer}

The bottom layer of the data management architecture is an instantiation of the data lake concept (Koumakis et al., 2018). A state of the art mobile health app for cancer management should have the capacity to connect with external applications, lifestyle sensors and devices. The vast amount of data currently generated and collected come in different streams and forms. These range from physician notes, personal health records, images from patient scans, health conversations in social media, continuous streaming information from wearables and other monitoring devices. For enabling a common representation of knowledge across the continuum of care and the different 
information sources, a platform needs to be in place to support information integration and semantic uplifting at runtime, complementing user profiles. Ontologies and semantics can play a key role, and big data management infrastructures can help in efficiently processing big amounts of information (Kondylakis et al., 2018).

Collecting, homogenizing and exploiting diverse, heterogeneous health data is essential for building mobile apps for cancer management that focus on personalization, medical recommendations and knowledge extraction (Kondylakis et al., 2019a). It consists of the data lake, data cleaning tools, the cleaned databases, semantic integration and mapping tools, a specific ontology, specific data management tools, application program interfaces (APIs) and security and anonymization tools:

- Data Lake: All inserted data are staged in a repository with multiple databases forming a data lake. Those databases can include biomedical, psychosocial, functional, demographic, psychological data and others and can be retrospective, prospective and external datasets. We have to note that datasets are completely anonymized before uploaded to the data lake (Vardalachakis et al., 2019). Besides having those datasets in their original form, there are also cleaned copies of the data in the data lake. Cleaning includes missing value handling, identification of erroneous records and others;

- Semantic Integration and Mapping: In order for the maximum exploitation of the information potential of these datasets, they should be homogenized, semantically uplifted and transparently accessed. To achieve this integration, a semantic model, such as a specific ontology, has been developed with the purpose of effectively represent and model all data. Having a semantic model available, the next step is to define the necessary mappings between the individual schemata and the semantic model in order to homogenize and integrate the various datasets. Those mappings provide declarative correspondences between the data columns and specific ontology terms. When those mappings are available, the data integration engine transforms, semantically uplifts and stores them in a central data warehouse. All data transformed into triples, will be served through the APIs via a query execution engine. In addition, for data series, a state of the art processing framework is used to provide effective and efficient access to those data (Kondylakis et al., 2019b);

- Data Access APIs: All data, both integrated and those residing in the data lake are accessible through RESTfull APIs (https://searchapparchitecture.techtarget. com/definition/RESTful-API);

- Security: As data access needs to be regulated this is ensured by an authentication and authorization server which is contacted prior to answering specific data requests, ensuring compliance with all necessary security and confidentiality requirements established by the security layer.

\section{Interoperability Issues}

One of the biggest challenges for healthcare providers is that patient data needed for the provision of the best treatment is often scattered across different systems rather 
than stored in a single location. For example, an oncologist may have another set of records than the one that a surgeon or a primary care provider may have. Technologies that facilitate care coordination through interoperability are improving, but seamless flow of information from one care setting to another still requires more progress. Interoperability is a primary consideration to achieve communication of applications, medical devices and health care providers (Kouroubali et al., 2019b). The growing demand for secondary use of clinical and administrative data renders the pressure towards solving interoperability challenges even more imperative (Kouroubali \& Katehakis, 2019).

The now commonplace use of Electronic Health Records (EHRs) has decreased some of the barriers when using medical records for research, although fully interoperable electronic medical record systems are not yet a wide reality. Several efforts for applying standards in the collection, extraction and integration of data by standardization bodies, governments, the research community and industry are in progress (Belenkaya et al., 2018) to establish and adopt clinically relevant and integrated standards covering the entire oncology sector. Organizations such as Health Level Seven International (HL7, http://www.hl7.org/) and Personal Connected Health Alliance (PCHAlliance, http://www.pchalliance.org/) Continua help towards the delivery of standards-based, open specifications that can support the flow of data from the point of capture into EHRs in the same format and coded content. Apps that are exploiting platforms and interoperability standards, such as SMART (https:// smarthealthit.org/) and Fast Healthcare Interoperability Resources (FHIR, http://www. h17.org/fhir/), specifically for cancer, have already started to emerge (Mandel et al., 2016; Warner et al., 2016). Integrating the Healthcare Enterprise (IHE, https://www. ihe.net/) describes how standards may be used effectively.

It is a fact that some data resolution may be lost during the process of mapping EHR fields to a formally described abstraction layer, but these common interfaces support queries across EHRs or the extraction of patient data in the same format to allow merging of patient sets between numerous institutions (Petrakis et al, 2019). In oncology, projects requesting EHRs to map fields, concepts, or other data to a formally described abstraction layer include, besides FHIR, the American Society of Clinical Oncology (ASCO) CancerLinQ (https://www.cancerlinq.org/), as well as ASCO mCODE (https://mcodeinitiative.org/).

\section{Security Considerations}

To ensure that privacy and personal data of patients are protected, the current European Union General Data Protection Regulation (GDPR) obligates data controllers to adopt data protection by design and by default approach in developing systems that process personal data. The GDPR is a regulation of general application and is principles-based. It includes the principle that the protection of personal data shall be a default property of personal data information systems. Controllers of personal data must put in place appropriate technical and organizational measures to implement the data protection principles, facilitate the enforcement of data subject rights, and secure the personal data 
under their care. A secure framework for the authorization, authentication, delegation, and auditing is mandatory for secure access and communication between services and patient interaction with the platform (Kondylakis et al., 2017b, Koumakis et al., 2018). A new promising technology for information and communication technology security and especially for the auditing mechanism is blockchain. The fundamental strength of a blockchain system lies in its data integrity and networked immutability (write once and read-only) digital events that can be applied in the healthcare domain (Kuo et al., 2017).

\section{Software Quality Assurance}

Software quality assurance consists of methods and tools to monitor the software engineering processes in order to ensure quality. The design and evaluation of any app should adhere to certain principles of systems and software engineering, such as the ones defined by International Organization for Standardization (ISO) 25000 series for systems and software quality requirements and evaluation (International Organization for Standardization, 2014). ISO 25000 has organized the requirement into eight main categories, and its respective sub-categories, including functional suitability, performance efficiency, compatibility, usability, reliability, security maintainability and portability. Quality assurance through quality labelling is very important to promote the design, development and implementation of functional and effective applications.

\section{DISCUSSION}

Health IT brings clinical data and patient information together and guides oncologists in making evidence-based care decisions that lead to better outcomes. The potential benefits of interoperable interconnected tools and health systems are particularly important for oncology (McCabe, 2019), as providing cancer-wide care depends on access to accurate and complete information, as well as extensive coordination between patients, caregivers, and diverse provider groups through treatment and survivorship. Connecting the EHR has the potential to support diagnosis assistance around complex patients (Gong et al., 2019; Katehakis \& Kouroubali, 2020).

The need for consolidation and standardization efforts to create interoperable solutions is timely and critical (Belenkaya et al., 2018), as well as the need for the cancer informatics community for national initiatives for data standardization and large-scale multi-disciplinary research collaborations. In addition to supporting cancer care, cancer-related standards will help improve surveillance and research. Similar to the migration from paper to electronic records, the shift toward data interoperability between EHR vendors may require policy changes before full adoption is possible (Sicotte et al., 2017; Chambers et al., 2019).

National infrastructures in Europe provide for horizontal electronic Government services (such as for electronic identification), as well as electronic services to support national health systems (e.g. for electronic Confirmation, Reimbursement, Prescription, 
and others) (Katehakis et al., 2018). Services are provided to both the public, as well as the private sector (i.e. primary care, hospitals, clinical centers, pharmacies, etc.). An integration platform, operating under specific interoperability agreements, may provide for (indicatively) certain home care, decision support, and research services.

Apps available cannot replace, e.g. an oncologist, but they can provide the means to make the painful process easier for patients and their families during the treatment. Apps do not intend to diagnose or treat cancer by themselves. Still, they can aid in early cancer diagnosis and treatment, provided they be tested and certified properly. For doing so, proper regulation is required and a framework to support interoperability at all levels (at home, at primary care, at the hospital, regionally, at a national or international level) for all relevant use cases (eHealth Network, 2015; Katehakis \& Kouroubali, 2019).

Limiting semantic fragmentation is a key challenge to address. Properly designed data (structure ad semantics) formats and secure communication APIs can successfully shield mobile app developers from complexity when integrating with proprietary vendor systems.

\section{Recommendations}

The following recommendations for building mobile applications for cancer patients need to be considered:

- Patient engagement is essential, right from the first steps of the application design. Employing participatory design will lead to an app that has been designed by the end-users for the end-users;

- An iterative design approach is required, incorporating user feedback until the device reaches an acceptable level of usability;

- The graphical user interface and usability are essential when building apps for patients. The interface should be simple, helping patients to focus on specific tasks without unnecessary menus and information;

- A mobile app should be personalized to the individual patient characteristics, such as presence of comorbidities, age, gender and others. Cancer is a multifaceted problem with many different treatment options and paths. Mobile apps offer the opportunity for learning from the data in order to provide intelligent personalized support;

- All information should be reliable, based on scientifically and clinically validated knowledge. Healthcare providers need to be engaged in the design and the development of the app;

- The app should comply with all protection of personal health information and security requirements, allowing the users to easily understand where their data are stored and who can use them, gradually building patient's trust. 
The proposed approach and integrated architecture for the development of highquality health apps for cancer do not replace medical practice but rather enhance and facilitate patient management.

Future research in apps for cancer patients need to focus on the challenges and issues related to security, privacy, and trust, and ways to help users gain confidence in adopting them. Other aspects for future study involve the improvement of user experience looking at usability, personalization, familiarity, and comfort.

As already proposed by (President's Cancer Panel, 2016) enabling interoperability among institutions and individuals that support care delivery across the cancer continuum - from prevention through treatment, survivorship, and end-of-life care - is considered to be essential. Doing so requires developing, testing, disseminating and adopting technical standards for information related to cancer care across the continuum in order to optimize the flow of information to serve the needs of patients, caregivers, and providers (Katehakis et al., 2017). For doing so, standard, open application programming interface (API) platforms should be developed and used to facilitate development of cancer-related apps.

As already mentioned by (European Commission, 2016), standards and protocols that aim to enhance interoperability of different data sets are a highly relevant field for policy action. The adoption of current technical standards, policies and best practices should be promoted, and new common ground and core definitions should be established (e.g. for data governance and usability to keep up with the rapidly changing technological environment, evidence and value, and analytical protocols). Incentives for the promotion of standards for the interoperability of clinical data, such as EHR or for genome experiment data, should be developed, promoted, and incentivized to allow for pooling of data and comparison of system-level research should be provided for the comparison of research outcomes.

\section{CONCLUSION}

The open use and sharing of Big Data, without compromising patients' rights to privacy and confidentiality, should be promoted.As cancer has been transformed into a chronic disease, cancer survival means living with a chronic and complex condition. Mobile technologies are becoming an integral part of daily life creating a great opportunity for effective empowerment of cancer patients. The vast number of parameters affecting the long-term management of the disease require integrated approaches to unleash the potential benefits of mobile apps. A holistic, integrated mobile app should focus on all areas of the cancer continuum, from prevention, surveillance, and intervention to consequences of cancer and its treatment, rehabilitation, and coordination between specialists and care providers. 
International Journal of Big Data and Analytics in Healthcare

Volume 5 - Issue $1 \cdot$ January-June 2020

\section{ACKNOWLEDGMENT}

Work reported in this chapter was partially supported by the Computational Biomedicine Lab \& the Center for eHealth Applications and Services, Institute of Computer Science, Foundation for Research and Technology - Hellas. 


\section{REFERENCES}

Basch, E., Deal, A. M., Kris, M. G., Scher, H. I., Hudis, C. A., Sabbatini, P., \& Schrag, D. et al. (2016). Symptom monitoring with patient-reported outcomes during routine cancer treatment: A randomized controlled trial. Journal of Clinical Oncology, 34(6), 557-565. doi:10.1200/JCO.2015.63.0830 PMID:26644527

Belenkaya, R., Campbell, W. S., Chen, R., Gurley, M. J., Huff, S. M., Randhawa, G., \& Rocca, M. et al. (2018). Data Standardization in Cancer: Challenges and Opportunities. AMIA.

Bray, F., Ferlay, J., Soerjomataram, I., Siegel, R. L., Torre, L. A., \& Jemal, A. (2018). Global cancer statistics 2018: GLOBOCAN estimates of incidence and mortality worldwide for 36 cancers in 185 countries. CA: a Cancer Journal for Clinicians, 68(6), 394-424. doi:10.3322/caac.21492 PMID:30207593

Chambers, D. A., Amir, E., Saleh, R. R., Rodin, D., Keating, N. L., Osterman, T. J., \& Chen, J. L. (2019). The Impact of Big Data Research on Practice, Policy, and Cancer Care. American Society of Clinical Oncology Educational Book, 39(39), e167-e175. doi:10.1200/EDBK_238057 PMID:31099675

Chatzimina, M., Koumakis, L., Marias, K., \& Tsiknakis, M. (2019, October). Employing Conversational Agents in Palliative Care: A Feasibility Study and Preliminary Assessment. In 2019 IEEE 19th International Conference on Bioinformatics and Bioengineering (BIBE), (pp. 489-496). IEEE.

Cognizant. (2019, October). Healthcare on Demand: New Provider Business Models for the Digital Economy. Retrieved from https://www.cognizant.com/whitepapers/ new-provider-business-models-for-the-digital-economy-codex4969.pdf

Denis, F., Lethrosne, C., Pourel, N., Molinier, O., Pointreau, Y., Domont, J., \& Letellier, C. et al. (2017). Randomized trial comparing a web-mediated follow-up with routine surveillance in lung cancer patients. Journal of the National Cancer Institute, 109(9).

eHealth Network. (2015). Refined eHealth European interoperability framework. Retrieved from https://ec.europa.eu/health//sites/health/files/ehealth/docs/ ev_20151123_co03_en.pdf

European Commission. (2016, Dec.). Study on Big Data in Public Health, Telemedicine and Healthcare. Final Report.

Girault, A., Ferrua, M., Lalloué, B., Sicotte, C., Fourcade, A., Yatim, F., \& Minvielle, E. et al. (2015). Internet-based technologies to improve cancer care coordination: Current use and attitudes among cancer patients. European Journal of Cancer, 51(4), 551-557. doi:10.1016/j.ejca.2014.12.001 PMID:25661828

Gong, M., Wang, Z., Liu, Y., Zhou, H., Wang, F., Wang, Y., \& Hong, N. (2019). Toward early diagnosis decision support for breast cancer: Ontology-based semantic interoperability. Academic Press. 
International Organization for Standardization. (2014). ISO/ IEC 25000:2014, Systems and software engineering -- Systems and software Quality Requirements and Evaluation (SQuaRE) -- Guide to SQuaRE. ISO/ IEC JTC 1/ SC 7, Software and systems engineering. Retrieved from https://www.iso.org/standard/64764.html

Katehakis, D. G., Kondylakis, H., Koumakis, L., Kouroubali, A., \& Marias, K. (2017). Integrated care solutions for the citizen: Personal health record functional models to support interoperability. EJBI, 13(1), 41-56. doi:10.24105/ejbi.2017.13.1.8

Katehakis, D. G., \& Kouroubali, A. (2019). A Framework for eHealth Interoperability Management. Journal of Strategic Innovation and Sustainability, 14(5).

Katehakis, D. G., \& Kouroubali, A. (2020). Digital Transformation Challenges for the Implementation of Quality Electronic Medical Records. In Quality Assurance in the Era of Individualized Medicine (pp. 63-91). IGI Global. doi:10.4018/978-1-79982390-2.ch003

Katehakis, D. G., Kouroubali, A., \& Fundulaki, I. (2018). Towards the Development of a National eHealth Interoperability Framework to Address Public Health Challenges in Greece. SWH@ ISWC.

Katehakis, D. G., \& Tsiknakis, M. (2006). Electronic Health Record. Wiley Encyclopedia of Biomedical Engineering. Hoboken, NJ: John Wiley \& Sons, Inc.

Kondylakis, H., Bucur, A., Dong, F., Renzi, C., Manfrinati, A., Graf, N., . . Kiefer, S. (2017a). iManageCancer: Developing a Platform for Empowering Patients and Strengthening Self-Management in Cancer Diseases. Computer-Based Medical Systems (CBMS), IEEE 30th International Symposium, 755-760.

Kondylakis, H., Dayan, N., Zoumpatianos, Z., \& Palpanas, T. (2019a). Coconut: Sortable summarizations for scalable indexes over static and streaming data series. The VLDB Journal, 28(6), 847-869. doi:10.1007/s00778-019-00573-w

Kondylakis, H., Dayan, N., Zoumpatianos, Z., \& Palpanas, T. (2019b). Coconut Palm: Static and Streaming Data Series Exploration Now in your Palm. SIGMOD Conference, 1941-1944. doi:10.1145/3299869.3320233

Kondylakis, H., Koumakis, L., Hänold, S., Nwankwo, I., Forgó, N., Marias, K., \& Graf, N. (2017b). Donor's support tool: Enabling informed secondary use of patient's biomaterial and personal data. International Journal of Medical Informatics, 97, 282-292. doi:10.1016/j.ijmedinf.2016.10.019 PMID:27919386

Kondylakis, H., Koumakis, L., Katehakis, D. G., Kouroubali, A., Marias, K., Tsiknakis, M., . . . Karademas, E. (2019). Developing a data infrastructure for enabling breast cancer women to BOUNCE back. In 2019 IEEE 32nd International Symposium on Computer-Based Medical Systems (CBMS) (pp. 652-657). IEEE. doi:10.1109/ CBMS.2019.00134 
Kondylakis, H., Koumakis, L., Tsiknakis, M., \& Marias, K. (2018). Implementing a data management infrastructure for big healthcare data. IEEE EMBS International Conference on Biomedical \& Health Informatics (BHI), 361-364. doi:10.1109/ BHI.2018.8333443

Kotronoulas, G., Kearney, N., Maguire, R., Harrow, A., Di Domenico, D., Croy, S., \& MacGillivray, S. (2014). What is the value of the routine use of patient-reported outcome measures toward improvement of patient outcomes, processes of care, and health service outcomes in cancer care? A systematic review of controlled trials. Journal of Clinical Oncology, 32(14), 1480-1501. doi:10.1200/JCO.2013.53.5948 PMID:24711559

Koumakis, L., Kondylakis, H., Katehakis, D. G., Iatraki, G., Argyropaidas, P., Hatzimina, M., \& Marias, K. (2018). A content-aware analytics framework for open health data. Precision Medicine Powered by pHealth and Connected Health, 59-64.

Kouroubali, A. (2004). Implementation of health care information systems: Key factors and the dynamics of change (Doctoral dissertation). University of Cambridge.

Kouroubali, A., \& Katehakis, D. G. (2019). The new European interoperability framework as a facilitator of digital transformation for citizen empowerment. Journal of Biomedical Informatics, 94, 103166. doi:10.1016/j.jbi.2019.103166 PMID:30978512

Kouroubali, A., Kondylakis, H., Koumakis, L., Papagiannakis, G., Zikas, P., \& Katehakis, D. G. (2019a). iSupport: Building a Resilience Support Tool for Improving the Health Condition of the Patient During the Care Path. Studies in Health Technology and Informatics, 261, 253-258. PMID:31156125

Kouroubali, A., Koumakis, L., Kondylakis, H., \& Katehakis, D. G. (2019b). An Integrated Approach Towards Developing Quality Mobile Health Apps for Cancer. In Mobile Health Applications for Quality Healthcare Delivery (pp. 46-71). IGI Global. doi:10.4018/978-1-5225-8021-8.ch003

Kouroubali, A., Starren, J., Barrows, R. C. Jr, \& Clayton, P. D. (1997). Practical lessons in remote connectivity. Proceedings of the AMIA Annual Fall Symposium, 335.

Kuo, T. T., Kim, H. E., \& Ohno-Machado, L. (2017). Blockchain distributed ledger technologies for biomedical and health care applications. Journal of the American Medical Informatics Association, 24(6), 1211-1220. doi:10.1093/jamia/ocx068 PMID:29016974

Kushi, L. H., Doyle, C., McCullough, M., Rock, C. L., Demark-Wahnefried, W., Bandera, E. V., \& Gansler, T. et al. (2012). American Cancer Society Guidelines on nutrition and physical activity for cancer prevention: Reducing the risk of cancer with healthy food choices and physical activity. CA: a Cancer Journal for Clinicians, 62(1), 30-67. doi:10.3322/caac.20140 PMID:22237782

LeBlanc, T. W., \& Abernethy, A. P. (2017). Patient-reported outcomes in cancer care-Hearing the patient voice at greater volume. Nature Reviews. Clinical Oncology, 14(12), 763-772. doi:10.1038/nrclinonc.2017.153 PMID:28975931 
Maher, E. J. (2013). Managing the consequences of cancer treatment and the English National Cancer Survivorship Initiative. Acta Oncologica, 52(2), 225-232. doi:10.3 109/0284186X.2012.746467 PMID:23237053

Mandel, J. C., Kreda, D. A., Mandl, K. D., Kohane, I. S., \& Ramoni, R. B. (2016). SMART on FHIR: A standards-based, interoperable apps platform for electronic health records. Journal of the American Medical Informatics Association, 23(5), 899-908. doi:10.1093/jamia/ocv189 PMID:26911829

Manikis, G. C., Kourou, K., Poikonen-Saksela, P., Kondylakis, H., Karademas, E., Marias, K., . . . Fotiadis, D. I. (2019, October). Computational modeling of psychological resilience trajectories during breast cancer treatment. In 2019 IEEE 19th International Conference on Bioinformatics and Bioengineering (BIBE), (pp. 423-427). IEEE.

Maramis, C., Karamanidou, C., Schera, F., Kiefer, S., Koumakis, L., Marias, K., . . . Pospisilova, S. (2019). Using Electronic Patient Reported Outcomes to Foster Palliative Cancer Care: The MyPal Approach. In 2019 IEEE 19th International Conference on Bioinformatics and Bioengineering (BIBE), (pp. 405-409). IEEE.

Martínez-Pérez, B., De La Torre-Díez, I., \& López-Coronado, M. (2015). Privacy and security in mobile health apps: A review and recommendations. Journal of Medical Systems, 39(1), 181. doi:10.1007/s10916-014-0181-3 PMID:25486895

McCabe, R. M. (2019, October). National cancer database: The past, present, and future of the cancer registry and its efforts to improve the quality of cancer care. Seminars in Radiation Oncology, 29(4), 323-325. doi:10.1016/j.semradonc.2019.05.005 PMID:31472733

McGuire, S. (2016). World Cancer Report 2014. Geneva, Switzerland: World Health Organization, International Agency for Research on Cancer, WHO Press, 2015. Advances in Nutrition: An International Review Journal, 7(2), 418-419.

Pai, A. L., Patiño-Fernández, A. M., McSherry, M., Beele, D., Alderfer, M. A., Reilly, A. T., \& Kazak, A. E. et al. (2007). The Psychosocial Assessment Tool (PAT2. 0 ): Psychometric properties of a screener for psychosocial distress in families of children newly diagnosed with cancer. Journal of Pediatric Psychology, 33(1), 50-62. doi:10.1093/jpepsy/jsm053 PMID:17609228

Petrakis, Y., Kouroubali, A., \& Katehakis, D. (2019). A Mobile App Architecture for Accessing EMRs Using XDS and FHIR. In 2019 IEEE 19th International Conference on Bioinformatics and Bioengineering (BIBE), (pp. 278-283). IEEE.

President's Cancer Panel. (2016). Improving Cancer-Related Outcomes with Connected Health: A Report to the President of the United States from the President's Cancer Panel. Bethesda, MD: President's Cancer Panel. Retrieved from https://prescancerpanel. cancer.gov/report/connectedhealth/ 
Rincon, E., Monteiro-Guerra, F., Rivera-Romero, O., Dorronzoro-Zubiete, E., SanchezBocanegra, C. L., \& Gabarron, E. (2017). Mobile Phone Apps for Quality of Life and Well-Being Assessment in Breast and Prostate Cancer Patients: Systematic Review. JMIR mHealth and uHealth, 5(12), e187. doi:10.2196/mhealth.8741 PMID:29203459

Roehrs, A., Da Costa, C. A., Da Rosa Righi, R., \& De Oliveira, K. S. F. (2017). Personal health records: A systematic literature review. Journal of Medical Internet Research, 19(1), e13. doi:10.2196/jmir.5876 PMID:28062391

Sicotte, C., Clavel, S., \& Fortin, M. A. (2017). A cancer care electronic medical record highly integrated into clinicians' workflow: Users' attitudes pre-post implementation. European Journal of Cancer Care, 26(6), e12548. doi:10.1111/ ecc.12548 PMID:27454341

Stoyanov, S. R., Hides, L., Kavanagh, D. J., Zelenko, O., Tjondronegoro, D., \& Mani, M. (2015). Mobile app rating scale: A new tool for assessing the quality of health mobile apps. JMIR mHealth and uHealth, 3(1), e27. doi:10.2196/mhealth.3422 PMID:25760773

Triantafyllidis, A. K., Koutkias, V. G., Chouvarda, I., Adami, I., Kouroubali, A., \& Maglaveras, N. (2016). Framework of sensor-based monitoring for pervasive patient care. Healthcare Technology Letters, 3(3), 153-158. doi:10.1049/ht1.2016.0017 PMID:27733920

US Food and Drug Administration. (2006). Guidance for industry: patient-reported outcome measures: use in medical product development to support labelling claims: draft guidance. Health and Quality of Life Outcomes, 4(79).

Valderas, J. M., Kotzeva, A., Espallargues, M., Guyatt, G., Ferrans, C. E., Halyard, M. Y., \& Alonso, J. et al. (2008). The impact of measuring patient-reported outcomes in clinical practice: A systematic review of the literature. Quality of Life Research: An International Journal of Quality of Life Aspects of Treatment, Care and Rehabilitation, 17(2), 179-193. doi:10.1007/s11136-007-9295-0 PMID:18175207

Vardalachakis, M., Kondylakis, H., Koumakis, L., Kouroubali, A., \& Katehakis, D. G. (2019). ShinyAnonymizer: A tool for anonymizing health data. In International Conference on Information and Communication Technologies for Ageing Well and $e$-Health. ICT4AWE. doi:10.5220/0007798603250332

Warner, J. L., Rioth, M. J., Mandl, K. D., Mandel, J. C., Kreda, D. A., Kohane, I. S., \& Yao, H. et al. (2016). SMART precision cancer medicine: A FHIR-based app to provide genomic information at the point of care. Journal of the American Medical Informatics Association, 23(4), 701-710. doi:10.1093/jamia/ocw015 PMID:27018265

Wiljer, D., Leonard, K. J., Urowitz, S., Apatu, E., Massey, C., Quartey, N. K., \& Catton, P. (2010). The anxious wait: Assessing the impact of patient accessible EHRs for breast cancer patients. BMC Medical Informatics and Decision Making, 10(1), 46. doi:10.1186/1472-6947-10-46 PMID:20809950 
Yaqoob, I., Ahmed, E., Hashem, I. A. T., Ahmed, A. I. A., Gani, A., Imran, M., \& Guizani, M. (2017). Internet of Things architecture: Recent advances, taxonomy, requirements, and open challenges. IEEE Wireless Communications, 24(3), 10-16. doi:10.1109/MWC.2017.1600421 
Angelina Kouroubali $(P h D)$ is a Collaborating Researcher at the Computational BioMedicine Laboratory and a research expert at the Centre of eHealth Applications and Services at the Institute of Computer Science, Foundation for Research and Technology-Hellas (FORTH). She holds a Ph.D. in Management of Medical Informatics from the Judge Business School, University of Cambridge, a Masters in Medical Informatics from Columbia University, NY, USA, and a Bachelors degree in Biology from Bard College, USA. Dr. Kouroubali has more than 20 years of professional experience in the field of Medical Informatics.

Lefteris Koumakis $(P h D)$ is a postdoctoral researcher with the Computational BioMedicine Laboratory of the Foundation for Research and Technology Hellas (FORTH). He received his PhD degree in Computational Biology from the Technical University of Crete. He is biomedical informatics professional with expertise in computational biology, bioinformatics, data mining and software infrastructures for life sciences. He is the IT Architect of the Greek Biobanks network (BBMR-GR), responsible for the coordination and connection of the Greek biobank Centers and their linkage with collections in other European Center via the BBMRI-ERIC. He has extensive experience in participating and leading more than 15 European Projects and has published many papers in scientific journals, proceedings of international conferences \& workshops, related to his fields of expertise. 
Haridimos Kondylakis is currently a Collaborating Researcher at Computational BioMedicine Laboratory (CBML), Institute of Computer Science, Foundation of Research \& Technology-Hellas (FORTH). He holds a Ph.D and an M.Sc. in Computer Science from University of Crete. He is also a visiting lecturer at the Computer Science Department, University of Crete and at the Department of Electric and Computer Engineeringat Hellenic Mediterranean University and at Hellenic Open University, teaching lessons related to data management and semantics. His interests include semantic-based, big data management topics and their application in healthcare. More specifically he has focused on e-Consent through semantic rules, semantically-enabled medical recommendations for patient empowerment, ontology summarization and evolution, efficient infrastructures for collecting, partitioning, indexing, integrating and managing eHealth information and data series data, personal health environments, modular ontologies for healthcare and mappings to the various data sources. In the past he has participated in more than 15 EU research projects with a total budget over 60Mie and he is currently participating in two H2020 EU (BOUNCE - WP Leader \& STARS) research projects. He has more than 130 publications in international conferences, books and journals including ACM SIGMOD, VLDB, JWS, KER, EDBT, ISWC, ESWC etc. He is the lead guest editor of the special issue for PHRs at the JBI journal, the publicity chair of ISWC 2020, the organizer of the International Workshop on Semantic Web Technologies for Health Data Management, the organizational chair of ISIP 2019 conference and the special session chair of BIBE 2019. He also acts as a regular reviewer and a PC member for a number of premier journals and conferences, such as JWS, JODS, CIKM, EDBT, ISWC, etc.

Dimitrios Katehakis holds an Electrical Engineering Diploma from the Technical University of Patras, Greece, and an MS in Electrical Engineering, from the University of Maryland at College Park. He heads the Center for eHealth Applications and Services at FORTH-ICS since 2010. He is also the Secretary General of HL7 Hellas (since 2014), and the Hellenic Health Informatics Association (since 2018). He has participated in the implementation of a large number of eHealth projects, in both Europe and the US. His work focuses on data integration analysis and the design of information systems and distributed architectures for forward-looking, integrated EHR services. Products of his work operate in a large number of health units in Greece. Areas of expertise: eHealth, EHR standards, EHR systems, Health IT Solutions, HL7, Hospital Information Systems, Interoperability. 